Document downloaded from:

http://hdl.handle.net/10251/50839

This paper must be cited as:

Fakharany, M.; Company Rossi, R.; Jódar Sánchez, LA. (2014). Positive finite difference schemes for a partial integro-differential option pricing model. Applied Mathematics and Computation. 249:320-332. doi:10.1016/j.amc.2014.10.064.

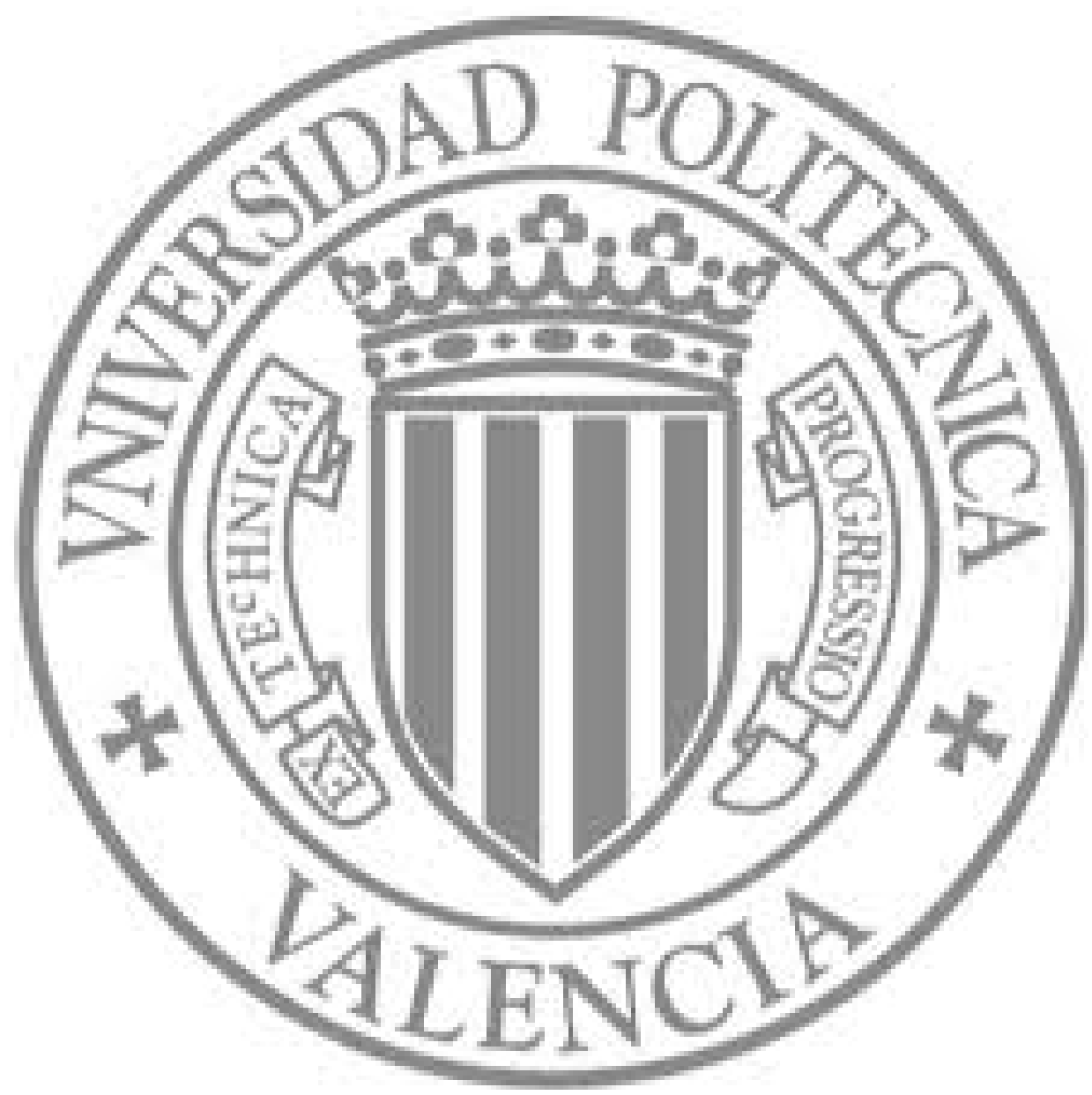

The final publication is available at

http://dx.doi.org/10.1016/j.amc.2014.10.064

Copyright Elsevier 


\title{
Positive finite difference schemes for a partial integro-differential option pricing model
}

\author{
M. Fakharany, R. Company ${ }^{1}$, L. Jódar \\ Instituto de Matemática Multidisciplinar, Universitat Politècnica de València, Camino de \\ Vera $s / n$, 46022 Valencia, Spain
}

\begin{abstract}
This paper provides a numerical analysis for European options under partial integro-differential Bates model. An explicit finite difference scheme has been used for the differential part, while the integral part has been approximated using the four-points open type formula. The stability and consistency have been studied. Moreover, conditions guaranteing positivity of the solutions are provided. Illustrative numerical examples are included.

Keywords: Partial integro-differential equation, Bates model, numerical analysis, stability and positivity.
\end{abstract}

\section{Introduction}

It is well known that the geometric Brownian motion proposed by BlackScholes [1] fails to reflect some empirical phenomena such as the volatility smiles and skews in the return distribution and the large random fluctuations as crashes

5 and rallies. There are two ways of developing option pricing models capturing such behavior; firstly adding jumps into the price process for the underlying asset, as proposed by Merton [2] and Kou [3]; secondly, allowing the volatility to evolve stochastically for instant Hull and White [4] and Heston [5].

\footnotetext{
Email addresses: fakharany@aucegypt.edu (M. Fakharany), rcompany@imm.upv.es (R. Company), ljodar@imm.upv.es (L. Jódar)

${ }^{1}$ corresponding author
}

Preprint submitted to Applied Mathematics and Computation

October 14, 2014 
Essentially, stochastic volatility appears to be needed to explain the variation in strike at longer time, although it performs poorly across different maturities, especially at shorter time. Adding jumps to the price and/or the volatility provides a great flexibility allowing to explain the strike variation at shorter time, 6, Chap. 14]. In this sense, Cont and Tankov (2003) indicate that a 15 model combining both stochastic volatility and jump diffusion feather provides more reasonable results.

Bates Model [8] combines the Merton and Heston models by adding lognormally distributed jumps to the square root volatility process introduced by 20 Heston. Other further extensions have been studied in [9, 10, 11.

In this paper we deal with the Bates model that describes the behavior of the underlying asset $S$ and its variance $\nu$ by the coupled stochastic differential equations:

$$
\begin{gathered}
d S(t)=(r-q-\lambda \xi) S(t) d t+\sqrt{\nu(t)} S(t) d W_{1}+(\eta-1) S(t) d Z(t), \\
d \nu(t)=\kappa(\theta-\nu(t)) d t+\sigma \sqrt{\nu(t)} d W_{2}, \\
d W_{1} d W_{2}=\rho d t,
\end{gathered}
$$

where $W_{1}$ and $W_{2}$ are standard Brownian motions, $Z$ is the poisson process. The parameter $r$ is the risk free interest rate, $q$ is the continuous dividend yield, $\lambda$ is the jump intensity, $\kappa$ is the mean reversion rate, $\theta$ is the long-run variance, $\sigma$ is the volatility of the variance $\nu, \rho$ is the Wiener correlation parameter, $\eta$ is the jump amplitude of the jump diffusion process and $\xi$ is the expected relative jump size $(\xi=E[\eta-1])$. By using Itô calculus and standard arbitrage arguments one gets the partial integro-differential equation (PIDE) with the unknown option price $U(S, \nu, \tau)$ [12, 13 ]

$$
\begin{gathered}
\frac{\partial U}{\partial \tau}=\frac{1}{2} \nu S^{2} \frac{\partial^{2} U}{\partial S^{2}}+\rho \sigma \nu S \frac{\partial^{2} U}{\partial S \partial \nu}+\frac{1}{2} \sigma^{2} \nu \frac{\partial^{2} U}{\partial \nu^{2}}+(r-q-\lambda \xi) S \frac{\partial U}{\partial S}+\kappa(\theta-\nu) \frac{\partial U}{\partial \nu}-(r+\lambda) U \\
+\lambda \int_{0}^{\infty} U(S \eta, \nu, \tau) f(\eta) d \eta
\end{gathered}
$$


where $\tau=T-t$ is the time to maturity. and the density function $f(\eta)$ is given by

$$
f(\eta)=\frac{1}{\sqrt{2 \pi} \hat{\sigma} \eta} \exp \left[-\frac{(\ln \eta-\mu)^{2}}{2 \hat{\sigma}^{2}}\right]
$$

where $\mu$ is the mean of the jump and $\hat{\sigma}$ is the standard deviation. For the European call option we consider the initial condition

$$
U(S, \nu, 0)=\max \{S-E, 0\}
$$

where $E$ is the strike price. We assume the boundary conditions applied to the Heston model, see [14, but modified for $\nu=0$ due to the additional integral term appearing in Bates model. For the boundaries $S=0$ and $S \rightarrow \infty$ one gets

$$
\begin{gathered}
U(0, \nu, \tau)=0, \\
\lim _{S \rightarrow \infty} \frac{\partial U}{\partial S}(S, \nu, \tau)=1 .
\end{gathered}
$$

Note that this last condition means a linear behavior of the option price for large values of $S$ with slope 1. Based on that fact, we replace it by the following condition, see [15, Chap. 3, pag. 54]

$$
U(S, \nu, \tau) \approx e^{-q \tau} S, \text { as } S \rightarrow \infty
$$

with slope $e^{-q \tau}$ for large values of $S$ due to the dividend payment. For $\nu \rightarrow \infty$ and $\nu=0$, the corresponding boundary conditions are imposed as follows

$$
\begin{gathered}
\lim _{\nu \rightarrow \infty} U(S, \nu, \tau)=S \\
\frac{\partial U}{\partial \tau}(S, 0, \tau)=(r-q-\lambda \xi) S \frac{\partial U}{\partial S}(S, 0, \tau)-(r+\lambda) U(S, 0, \tau)+\kappa \theta \frac{\partial U}{\partial \nu}(S, 0, \tau) \\
+\frac{\lambda}{\sqrt{2 \pi} \hat{\sigma}} \int_{0}^{\infty} U(\varphi, 0, \tau) \exp \left[-\frac{(\ln \varphi-\ln S-\hat{\mu})^{2}}{2 \hat{\sigma}^{2}}\right] \frac{d \varphi}{\varphi}
\end{gathered}
$$

where $\varphi=S \eta$.

25 Some authors used an alternative boundary condition see [21, 23]. Chiarella et. 
al. 13] used the method of lines to solve the American call option problem for Bates model by discretizing with respect to time and variance variables obtaining a system of first order ordinary differential equations with two unknowns the price and its derivative with respect to asset variable. Then the system so is solved using Riccati transformation, see [16]. Final discretization achieves a seven points stencil scheme treated using a linear complementarity problems (LCP). More recently [17] treat also the American call option problem under the Bates model using a full discretization for the spatial variable driving to a seven point finite difference stencil and the quadrature term is discretized using the quadrature rule based on piecewise linear interpolation. The authors use Rannacher scheme [18] for the time-stepping and the resulting LCP problem is solved using an iterative method.

The model (1)-(7) has two challenges from the numerical analysis point of view. Firstly, the presence of a mixed spatial derivative term involves the existence of 40 negative coefficient terms into the numerical scheme deteriorating the quality of the numerical solution such as spurious oscillation and slow convergence, see the introduction of [19]. Secondly, the discretization of the improper integral part should be adequate with the bounded numerical domain and the incorporation of the initial and boundary conditions.

45

Dealing with prices, guaranty of the positivity of the solutionis is essential. In this paper we construct an explicit difference scheme that guarantees positive solutions. We transform the PIDE (1) into a new PIDE without mixed spatial derivative before the discretization, following the idea of [20], and avoiding the above quoted drawbacks. Furthermore, this strategy has additional computational advantage of the reduction of the stencil scheme points, from nine [21, 22] or seven [13, 17] to just five.

The discrete treatment of the integral part is performed taking into account 55 the chosen boundary numerical domain together with the boundary conditions and using a composite four points integration formula of open type because of 
the higher order approximation of this rule [24, pp. 92-93].

The organization of the paper is as follows. In Section 2 we transform the original problem into a new one without cross derivative term. We also construct the difference scheme including its matrix form that will be used in Section 3 to study positivity and stability. Section 4 is addressed to the study of consistency of the scheme. Numerical examples illustrating the results for Bates European option model are included in Section 5. In Section 6, we consider the Bates model for American option using our finite difference scheme including the comparison with results of other authors.

Here we recall some useful definitions starting with the definition of the norm for vectors and matrices. For a given vector $\mathbf{v} \in \mathbb{R}^{n}$ such that $\mathbf{v}=\left(v_{1}, v_{2}, \ldots, v_{n}\right)^{T}$, the infinite norm of $\mathbf{v}$ is denoted by $\|\mathbf{v}\|_{\infty}$ and is defined as $\|\mathbf{v}\|_{\infty}=\max \left\{v_{j}, 1 \leq\right.$ $j \leq n\}$. The vector $\mathbf{v}$ is said to be nonnegative if $v_{j} \geq 0$ for all $1 \leq j \leq n$, then we denote $\mathbf{v} \geq 0$. For a matrix $B=\left(b_{i j}\right)_{n \times m}$ in $\mathbb{R}^{m \times n}$, we denote by $\|B\|_{\infty}=\max _{1 \leq i \leq m}\left\{\sum_{j=1}^{n} \mid b_{i j} \|\right\}$. Consequently if $A$ is a block matrix with $n \times m$ block entries $A_{i j}$, then the infinite norm of $A$, see [25, Chap. 2],

$$
\|A\|_{\infty}=\max _{1 \leq i \leq m}\left\{\left\|\left[A_{i 1} A_{i 2} \ldots A_{i n}\right]\right\|_{\infty}\right\} .
$$

Matrix $A$ is said to be nonnegative if $a_{i j} \geq 0$ for all $1 \leq i \leq m, 1 \leq j \leq n$, and we denote $A \geq 0$. For $x \in \mathbb{R}$, the error function of $x$ is denoted by $\operatorname{erf}(x)$ and is given by [26, pag. 93]

$$
\operatorname{erf}(x)=\frac{2}{\sqrt{\pi}} \int_{0}^{x} e^{-t^{2}} d t
$$

\section{Problem Transformation and Scheme Construction}

\subsection{The transformation of the problem}

We begin this section by eliminating the mixed spatial derivative term of (1), inspired by the reduction of second order linear partial differential equation 
in two independent variables to canonical form, see [27, Chap. 3] and [20] for details. Let us consider the following transformation

$$
x=\tilde{\rho} \sigma \ln S ; y=\rho \sigma \ln S-\nu ; w(x, y, \tau)=e^{(r+\lambda) \tau} U(S, \nu, \tau),
$$

where $\tilde{\rho}=\sqrt{1-\rho^{2}}, 0<|\rho|<1$, obtaining the following transformed equation

$$
\frac{\partial w}{\partial \tau}=\frac{\tilde{\rho}^{2} \nu \sigma^{2}}{2}\left(\frac{\partial^{2} w}{\partial x^{2}}+\frac{\partial^{2} w}{\partial y^{2}}\right)+\hat{\delta} \frac{\partial w}{\partial x}+\tilde{\delta} \frac{\partial w}{\partial y}+I(w),
$$

with

$$
I(w)=\lambda \int_{0}^{\infty} w(x+\sigma \tilde{\rho} \ln \eta, y+\rho \sigma \ln \eta, \tau) f(\eta) d \eta
$$

where

$$
\hat{\delta}=\sigma \tilde{\rho}\left(\hat{\xi}-\frac{\nu}{2}\right), \tilde{\delta}=\sigma \rho\left(\hat{\xi}-\frac{\nu}{2}\right)-\kappa(\theta-\nu) \text { and } \hat{\xi}=r-q-\lambda \xi
$$

For the sake of convenience in the matching of the further discretization of the differential and integral parts of 100 , we consider now the substitution

$$
\phi=x+\sigma \tilde{\rho} \ln \eta
$$

Hence from 2 and (11) one gets

$$
I(w)=\frac{\lambda}{\sqrt{2 \pi} \hat{\sigma} \tilde{\rho} \sigma} \int_{-\infty}^{\infty} w(\phi, y+m(\phi-x), \tau) \exp \left[\frac{-1}{\hat{\sigma}^{2}}\left(\frac{\phi-x}{\sigma \tilde{\rho}}-\mu\right)^{2}\right] d \phi,
$$

where $m=\frac{\rho}{\tilde{\rho}}$. Note that from $[9$, we have

$$
y=m x-\nu .
$$

The initial and boundary conditions (3)-(7) are transformed into the corresponding conditions using (9) and (13).

$$
\begin{gathered}
w(x, y, 0)=\max \left\{e^{\frac{x}{\sigma \tilde{\rho}}}-E, 0\right\}, \\
\lim _{x \rightarrow-\infty} w(x, y, \tau)=0, \\
w(x, y, \tau) \approx \exp \left[\frac{x}{\sigma \tilde{\rho}}+(r-q+\lambda) \tau\right], x \rightarrow \infty,
\end{gathered}
$$




$$
\begin{gathered}
w(x, y, \tau) \approx \exp \left[\frac{x}{\sigma \tilde{\rho}}+(r+\lambda) \tau\right], m x-y \rightarrow \infty, \\
\frac{\partial w}{\partial \tau} \approx \sigma \tilde{\rho} \hat{\xi} \frac{\partial w}{\partial x}+(\sigma \rho \hat{\xi}-\kappa \theta) \frac{\partial w}{\partial y} \\
+\frac{\lambda}{\sqrt{2 \pi} \hat{\sigma} \tilde{\rho} \sigma} \int_{-\infty}^{\infty} w(\phi, m \phi-\nu, \tau) \exp \left[\frac{-1}{\hat{\sigma}^{2}}\left(\frac{\phi-x}{\sigma \tilde{\rho}}-\mu\right)^{2}\right] d \phi, \nu \rightarrow 0 .
\end{gathered}
$$

From [28, 29] a suitable bound for the underlying asset variable $S$ is available and generally accepted. In an analogous way, considering an admissible range of the variance $\nu$, we can identify a convenient-bounded numerical domain $\mathcal{R}=$ $\left[S_{1}, S_{2}\right] \times\left[\nu_{1}, \nu_{2}\right]$ in the $S-\nu$ plane. Under the transformation (9) as it is shown in [20] the rectangle $\mathcal{R}$ is transformed into the rhomboid $A B C D$ as shown in Fig. 1, where the sides are given by

$$
\begin{aligned}
& \overline{A B}=\left\{(x, y) \in \mathbb{R}^{2} \mid a \leq x \leq b, y=m x-\nu_{2}\right\}, \\
& \overline{B C}=\left\{(x, y) \in \mathbb{R}^{2} \mid x=b, y=m b-\nu, \nu_{1} \leq \nu \leq \nu_{2}\right\}, \\
& \overline{C D}=\left\{(x, y) \in \mathbb{R}^{2} \mid a \leq x \leq b, y=m x-\nu_{1}\right\}, \\
& \overline{D A}=\left\{(x, y) \in \mathbb{R}^{2} \mid x=a, y=m a-\nu, \nu_{1} \leq \nu \leq \nu_{2}\right\},
\end{aligned}
$$

where

$$
a=\sigma \tilde{\rho} \ln S_{1}, \quad b=\sigma \tilde{\rho} \ln S_{2} .
$$

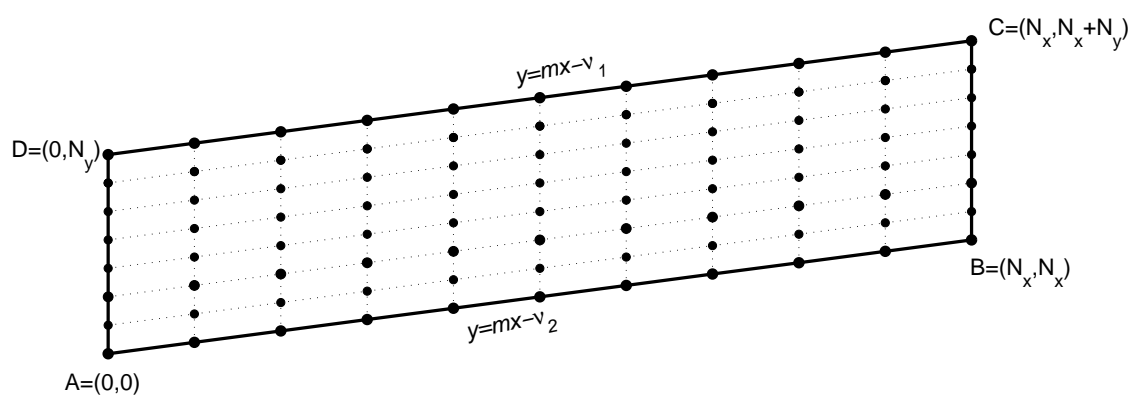

Fig. 1. Rhomboid numerical domain $A B C D$ 


\subsection{The numerical scheme}

In light of the transformation (9) and the boundary given by (21), we use a discretization of the numerical domain where the space stepsizes $h=\Delta x$ and $h_{y}=\Delta y=|m| h$ are related by the slope $m=\frac{\rho}{\tilde{\rho}}$. Here we subdivide space-time axes into uniform spaced points using

$$
\begin{gathered}
x_{i}=a+i h, 0 \leq i \leq N_{x}, \quad y_{j}=y_{0}+j|m| h, i \leq j \leq N_{y}+i, \\
\nu_{i, j}=m x_{i}-y_{j}, \quad \tau^{n}=n k, 0 \leq n \leq N_{\tau},
\end{gathered}
$$

where $h=\frac{b-a}{N_{x}}, y_{0}=m a-\nu_{2}, N_{y}=\frac{\nu_{2}-\nu_{1}}{|m| h}$ and $k=\frac{T}{N_{\tau}}$. Note that any mesh point in the computational spatial domain has the form

$$
\left(x_{i}, y_{j}\right)=\left(a+i h, m x_{i}-\nu_{2}+(j-i)|m| h\right) .
$$

The discretization for the boundary points is given by

$$
\begin{aligned}
& P(\overline{A B})=\left\{\left(x_{i}, y_{i}\right) \mid 0 \leq i \leq N_{x}\right\}, \\
& P(\overline{B C})=\left\{\left(x_{N_{x}}, y_{j}\right) \mid N_{x} \leq j \leq N_{x}+N_{y}\right\}, \\
& P(\overline{C D})=\left\{\left(x_{i}, y_{i+N_{y}}\right) \mid 0 \leq i \leq N_{x}\right\}, \\
& P(\overline{D A})=\left\{\left(x_{0}, y_{j}\right) \mid 0 \leq j \leq N_{y}\right\}
\end{aligned}
$$

Denote the approximate value of $w$ at a representative mesh point $P\left(x_{i}, y_{j}, \tau^{n}\right)$ by $W_{i, j}^{n}$, we implement the center difference approximation for spatial partial derivatives such that

$$
\begin{gathered}
\frac{\partial w}{\partial x} \approx \frac{W_{i+1, j}^{n}-W_{i-1, j}^{n}}{2 h} ; \frac{\partial w}{\partial y} \approx \frac{W_{i, j+1}^{n}-W_{i, j-1}^{n}}{2|m| h}, \\
\frac{\partial^{2} w}{\partial x^{2}} \approx \frac{W_{i+1, j}^{n}-2 W_{i, j}^{n}+W_{i-1, j}^{n}}{h^{2}} ; \frac{\partial^{2} w}{\partial y^{2}} \approx \frac{W_{i, j+1}^{n}-2 W_{i, j}^{n}+W_{i, j-1}^{n}}{m^{2} h^{2}},
\end{gathered}
$$

and $\frac{\partial w}{\partial \tau}$ is discretized using the explicit forward approximation

$$
\frac{\partial w}{\partial \tau} \approx \frac{W_{i, j}^{n+1}-W_{i, j}^{n}}{k} .
$$

For the approximation of the integral part $I(w)$ in 111 , the improper integral is truncated into $[a, b]$ and we implement the composite four points integration 
formula of open type [24, pp. 92-93] using the same step size for the variable $x$ as in the differential part. Hence the corresponding finite difference equation for 10 is given by

$$
\begin{gathered}
W_{i, j}^{n+1}=\beta_{i, j} W_{i, j}^{n}+\hat{\alpha}_{i, j} W_{i+1, j}^{n}+\breve{\alpha}_{i, j} W_{i-1, j}^{n}+\alpha_{i, j} W_{i, j-1}^{n}+\gamma_{i, j} W_{i, j+1}^{n}+\hat{\lambda} J_{i, j}^{n}, \\
1 \leq i \leq N_{x}-1, i+1 \leq j \leq N_{y}+i-1,0 \leq n \leq N_{\tau}-1
\end{gathered}
$$

where

$$
\begin{aligned}
& \beta_{i, j}=1-\frac{k \sigma^{2}}{h^{2} m^{2}} \nu_{i, j}=\left(1-\frac{k}{h^{2}} \tilde{a}_{i j}\right) \\
& \hat{\alpha}_{i, j}=\frac{k \sigma \tilde{\rho}}{2 h}\left[\frac{(2 \tilde{\rho} \sigma-h)}{2 h} \nu_{i, j}+\hat{\xi}\right]=\frac{k}{h}\left(\frac{\rho^{2}}{2 h} \tilde{a}_{i j}+\tilde{b}_{i j}\right) \\
& \breve{\alpha}_{i, j}=\frac{k \sigma \tilde{\rho}}{2 h}\left[\frac{(2 \tilde{\rho} \sigma+h)}{2 h} \nu_{i, j}-\hat{\xi}\right]=\frac{k}{h}\left(\frac{\rho^{2}}{2 h} \tilde{a}_{i j}-\tilde{b}_{i j}\right), \\
& \alpha_{i, j}=\frac{k}{2|m| h}\left[\left(\frac{\sigma^{2} \tilde{\rho}^{2}}{|m| h}+\frac{\sigma \rho}{2}-\kappa\right) \nu_{i, j}-\sigma \rho \hat{\xi}+\kappa \theta\right]=\frac{k}{h}\left(\frac{\tilde{\rho}^{2}}{2 h} \tilde{a}_{i j}-\frac{m}{|m|} \tilde{b}_{i j}+\tilde{c}_{i j}\right), \\
& \gamma_{i, j}=\frac{k}{2|m| h}\left[\left(\frac{\sigma^{2} \tilde{\rho}^{2}}{|m| h}-\frac{\sigma \rho}{2}+\kappa\right) \nu_{i, j}+\sigma \rho \hat{\xi}-\kappa \theta\right]=\frac{k}{h}\left(\frac{\tilde{\rho}^{2}}{2 h} \tilde{a}_{i j}+\frac{m}{|m|} \tilde{b}_{i j}-\tilde{c}_{i j}\right) \\
& \hat{\lambda}=\frac{5 k h \lambda}{24 \sqrt{2 \pi} \hat{\sigma} \tilde{\rho} \sigma}
\end{aligned}
$$

and the integral part is given by

$$
\begin{aligned}
J_{i, j}^{n}= & \sum_{\ell=0}^{N_{x} / 5-1}\left(11 g_{i, 5 \ell+1} W_{5 \ell+1,5 \ell+1+j-i}^{n}+g_{i, 5 \ell+2} W_{5 \ell+2,5 \ell+2+j-i}^{n}\right. \\
& \left.+g_{i, 5 \ell+3} W_{5 \ell+3,5 \ell+3+j-i}^{n}+11 g_{i, 5 \ell+4} W_{5 \ell+4,5 \ell+4+j-i}^{n}\right),
\end{aligned}
$$

assuming that $N_{x}$ has been previously chosen as a multiple of 5 . The weight function $g_{i, \ell}$ is given by

$$
g_{i, \ell} \equiv g\left(x_{i}, \phi_{\ell}\right)=\exp \left[\frac{-1}{2 \hat{\sigma}^{2}}\left(\frac{\phi_{\ell}-x_{i}}{\sigma \tilde{\rho}}-\mu\right)^{2}\right], 0 \leq \ell \leq N_{x} .
$$


The initial condition (16) is discretized into

$$
W_{i, j}^{0}=\max \left\{\exp \left(\frac{x_{i}}{\sigma \tilde{\rho}}\right)-E, 0\right\}, 0 \leq i \leq N_{x}, i \leq j \leq N_{y}+i,
$$

and the two Dirichlet conditions $(17)$ along $\overline{A D}$ and $(18)$ along $\overline{A B}$ take the forms

$$
\begin{gathered}
W_{0, j}^{n}=0,0 \leq j \leq N_{y}-1,1 \leq n \leq N_{\tau}, \\
W_{i, i}^{n}=\exp \left[\frac{x_{i}}{\sigma \tilde{\rho}}+(r+\lambda) \tau^{n}\right], 1 \leq i \leq N_{x}, 1 \leq n \leq N_{\tau}
\end{gathered}
$$

respectively. For the boundary condition along $\overline{B C}, x$ is constant $x=b$ and from 18$)$ one gets

$$
W_{N_{x}, j}^{n}=\exp \left[\frac{b}{\sigma \tilde{\rho}}+(r+\lambda-q) \tau^{n}\right], N_{x}+1 \leq j \leq N_{x}+N_{y}, 1 \leq n \leq N_{\tau},
$$

Note that the boundary condition 20 along the oblique segment $\overline{C D}$ involves $\frac{\partial w}{\partial x}$ and $\frac{\partial w}{\partial y}$. By the way the spatial directional derivative of $w$ for fixed $\tau$ along the direction $\overline{C D}$ with unitary vector $\hat{\mathbf{u}}=(\tilde{\rho}, \rho, 0)$ is given by

$$
D_{\hat{\mathbf{u}}} w=\nabla w \cdot \hat{\mathbf{u}}=\tilde{\rho} \frac{\partial w}{\partial x}+\rho \frac{\partial w}{\partial y} .
$$

The centered finite difference approximation for the directional derivative along $C D$ at the mesh point $\left(x_{i}, y_{N_{y}+i}, \tau^{n}\right) \in P(\overline{C D})$ is given by

$$
D_{\hat{\mathbf{u}}} w \approx \frac{\tilde{\rho}}{2 h}\left(W_{i+1, N_{y}+i+1}^{n}-W_{i-1, N_{y}+i-1}^{n}\right),
$$

and the backward difference approximation has been used for the term $\kappa \theta \frac{\partial w}{\partial y}$,

$$
\kappa \theta \frac{\partial w}{\partial y} \approx \frac{\kappa \theta}{|m| h}\left(W_{i, N_{y}+i}^{n}-W_{i, N_{y}+i-1}^{n}\right),
$$

while the integral part of 20 is approximated using four points open type formula. For the sake of positivity of the coefficients of the scheme, we take the following special approximation of the term $\frac{\partial w}{\partial \tau}$

$$
\frac{\partial w}{\partial \tau} \approx \frac{1}{k}\left(W_{i, N_{y}+i}^{n+1}-\frac{1}{3}\left(W_{i-1, N_{y}+i-1}^{n}+W_{i, N_{y}+i}^{n}+W_{i+1, N_{y}+i+1}^{n}\right)\right) .
$$


From (37)-39 the boundary condition 20 is approximated by

$W_{i, N_{y}+i}^{n+1}=\hat{a}_{1} W_{i-1, N_{y}+i-1}^{n}+\hat{a}_{2} W_{i, N_{y}+i-1}^{n}+\hat{a}_{3} W_{i, N_{y}+i}^{n}+\hat{a}_{4} W_{i+1, N_{y}+i+1}^{n}+\hat{\lambda} J_{i, N_{y}+i}^{n}$,

for $1 \leq i \leq N_{x}-1$ and $0 \leq n \leq N_{\tau}-1$, where

$$
\hat{a}_{1}=\frac{1}{3}-\frac{k \sigma \tilde{\rho} \hat{\xi}}{2 h}, \hat{a}_{2}=\frac{k \kappa \theta}{|m| h}, \hat{a}_{3}=\frac{1}{3}-\hat{a}_{2}, \hat{a}_{4}=\frac{1}{3}+\frac{k \sigma \tilde{\rho} \hat{\xi}}{2 h}
$$

and $J_{i, N_{y}+i}^{n}$ is obtained from (31) taking $j=N_{y}+i$.

In order to study the stability of the numerical scheme (28)- 41 , let us write it in a matrix form. It is convenient to write the numerical solutions $\left\{W_{i, j}^{n}\right\}$ in a suitable vector form, following the strategy of [30, let us define the vector $\mathbf{W}^{n} \in \mathbb{R}^{\left(N_{x}+1\right)\left(N_{y}+1\right)}$ such that

$$
\mathbf{W}^{n}=\left[\begin{array}{llll}
\mathcal{W}_{0}^{n} & \mathcal{W}_{1}^{n} & \ldots & \mathcal{W}_{N_{x}}^{n}
\end{array}\right]^{T},
$$

where $\mathcal{W}_{i}^{n}$ are vectors in $\mathbb{R}^{\left(N_{y}+1\right)}$

$$
\mathcal{W}_{i}^{n}=\left[\begin{array}{llll}
W_{i, i}^{n} & W_{i, i+1}^{n} & \ldots & W_{i, i+N_{y}}^{n}
\end{array}\right] .
$$

Hence numerical scheme (28)- 41 can be written in a matrix form as

$$
\mathbf{W}^{n+1}=(\mathcal{D}+\mathcal{P}) \mathbf{W}^{n}, 0 \leq n \leq N_{\tau}-1,
$$

where $\mathcal{D}$ and $\mathcal{P}$ are square matrices of size $\left(N_{x}+1\right)\left(N_{y}+1\right) \times\left(N_{x}+1\right)\left(N_{y}+1\right)$ representing the discretization of the differential and integral parts of the scheme (28)-41] respectively. The block matrix $\mathcal{D}$ can be written in the explicit form

$$
\mathcal{D}=\left[\begin{array}{ccccccc}
I & \Theta & \Theta & \Theta & \ldots & \ldots & \Theta \\
\breve{C}(1) & B(1) & \hat{C}(1) & \Theta & \ldots & \ldots & \Theta \\
\Theta & \breve{C}(2) & B(2) & \hat{C}(2) & \Theta & \ldots & \Theta \\
\vdots & \Theta & \ddots & \ddots & \ddots & \vdots & \vdots \\
\vdots & \vdots & \ldots & \ldots & \breve{C}\left(N_{x}-1\right) & B\left(N_{x}-1\right) & \hat{C}\left(N_{x}-1\right) \\
\Theta & \Theta & \ldots & \ldots & \ldots & \Theta & e^{(r-q+\lambda) k} I
\end{array}\right]
$$


where $I$ and $\Theta$ are the identity and zero matrices in $\mathbb{R}^{\left(N_{y}+1\right) \times\left(N_{y}+1\right)}$. The block entries $\breve{C}(\ell), B(\ell)$ and $\hat{C}(\ell)$ are matrices $\in \mathbb{R}^{\left(N_{y}+1\right) \times\left(N_{y}+1\right)}$ such that

$$
\begin{aligned}
& \breve{c}_{i j}(\ell)=\left\{\begin{aligned}
\breve{\alpha}_{\ell, \ell+i-1}, & i=2, \ldots, N_{y}, j=i+1, \\
\hat{a}_{1}, & i=j=N_{y}+1, \\
0, & \text { otherwise. }
\end{aligned}\right. \\
& b_{i j}(\ell)=\left\{\begin{aligned}
e^{(\lambda+r) k}, & i=j=1, \\
\alpha_{\ell, \ell+i-1}, & j=i-1, i=2, \ldots, N_{y}, \\
\beta_{\ell, \ell+i-1}, & j=i, i=2, \ldots, N_{y}, \\
\gamma_{\ell, \ell+i-1}, & j=i+1, i=2, \ldots, N_{y}, \\
\hat{a}_{2}, & i=N_{y}+1, j=N_{y}, \\
\hat{a}_{3}, & i=j=N_{y}+1, \\
0, & \text { otherwise. }
\end{aligned}\right. \\
& \hat{c}_{i j}(\ell)=\left\{\begin{aligned}
\hat{\alpha}_{\ell, \ell+i-1}, & i=2, \ldots, N_{y}, j=i-1, \\
\hat{a}_{4}, & i=j=N_{y}+1, \\
0, & \text { otherwise. }
\end{aligned}\right.
\end{aligned}
$$

With respect to the matrix $\mathcal{P}$, we denote its block entries by $\mathcal{P}_{\ell s}$ such that

$$
\mathcal{P}_{\ell s}=\left\{\begin{aligned}
\Theta, & \ell=1 \text { and } N_{x}+1, \text { for } s=1, \ldots, N_{x}+1, \\
P^{(s)}(\ell-1), & \ell=2, \ldots, N_{x}, s=1, \ldots, N_{x}+1,
\end{aligned}\right.
$$

where $P^{(s)}(\ell-1)$ are matrices in $\mathbb{R}^{\left(N_{y}+1\right) \times\left(N_{y}+1\right)}$ their elements are denoted by $P_{i j}^{s}(\ell-1)$. Note that from the periodic weight structure $(\{0,11,1,1,11,0, \ldots\})$ of four points open type formula (31), one gets

$$
P^{(s)}(\ell-1)=\Theta, s=1,6, \ldots, N_{x}+1,
$$

for $s=2,7, \ldots, N_{x}-3$ and $s=5,10, \ldots, N_{x}$, we have

$$
P_{i j}^{(s)}(\ell-1)=\left\{\begin{aligned}
11 \hat{\lambda} g_{\ell-1, s-1}, & i=2,3, \ldots, N_{y}, N_{y}+1, i=j \\
0, & \text { otherwise. }
\end{aligned}\right.
$$

Finally for $s=3,8, \ldots, N_{x}-2$ and $s=4,9, \ldots, N_{x}-1$,

$$
P_{i j}^{(s)}(\ell-1)=\left\{\begin{array}{cl}
\hat{\lambda} g_{\ell-1, s-1}, & i=2,3, \ldots, N_{y}, N_{y}+1, i=j \\
0, & \text { otherwise. }
\end{array}\right.
$$


Thus the matrix representation of the scheme (31)- 41) has been detailed in (43.51).

\section{Numerical properties of the scheme}

\subsection{Positivity of the solution}

We start this section by providing suitable conditions on the step sizes that guarantee the positivity of the numerical solution $\left\{W_{i, j}^{n}\right\}$ of scheme $281-(41)$.

First let us present the following lemma

lemma 1. Let $f(z)=\frac{z}{|\alpha z+\beta|}, z \in I=\left[z_{1}, z_{2}\right]$ and $\alpha \beta \neq 0$ then the minimum of $f(z)$ in $0<z_{1} \leq z \leq z_{2}$ is achieved in one of the extremum of $I$, i.e.,

$$
\min _{z \in I} f(z)=\min \left\{\frac{z_{i}}{\left|\alpha z_{i}+\beta\right|}, i=1,2\right\} .
$$

Proof. If $\alpha z+\beta \neq 0$ for all $z_{1}<z<z_{2}$, then $f(z)$ is a monotonic function, consequently 52 holds. Otherwise there exists a value $z_{0}=\frac{-\beta}{\alpha}$ such that $f(z)$ is increasing in $\left[z_{1}, z_{0}[\right.$ and decreasing in $\left.] z_{0}, z_{2}\right]$ and then $(52)$ also holds true.

Note that as $\nu_{i, j}$ defined in 23) satisfy $0<\nu_{1} \leq \nu_{i, j} \leq \nu_{2}$, the coefficient $\beta_{i, j}$ of 29$]$ is nonnegative under the following condition

$$
\frac{k}{h^{2}}<\frac{m^{2}}{\sigma^{2} \nu_{2}} .
$$

Note also from 29p that coefficients $\hat{\alpha}_{i, j}$ and $\breve{\alpha}_{i, j}$ are simultaneously nonnegative provided that

$$
\left|\tilde{b}_{i j}\right| \leq \frac{\rho^{2}}{2 h} \tilde{a}_{i j}
$$

If $\tilde{b}_{i j}=0$, then 54 holds for any value of the step size $h$. Otherwise 54 can be written in the following form

$$
h \leq \frac{2 \sigma \tilde{\rho} \nu_{i, j}}{\left|2 \hat{\xi}-\nu_{i, j}\right|},
$$


and from lemma 1 for $z=\nu_{i, j}, \alpha=-1$ and $\beta=2 \hat{\xi}, z_{i}=\nu_{i}, i=1,2$, one gets that 55 is verified under condition

$$
h \leq h_{1}=\min \left\{\frac{2 \sigma \tilde{\rho} \nu_{i}}{\left|2 \hat{\xi}-\nu_{i}\right|}, i=1,2\right\} .
$$

Similarly, one guarantees the simultaneous positivity of the coefficients $\alpha_{i, j}$ and $\gamma_{i, j}$ under the condition

$$
h \leq \frac{\tilde{\rho}^{2} \sigma^{2} \nu_{i, j}}{2 m^{2}\left|\frac{|m|}{m} \tilde{b}_{i j}-\tilde{c}_{i j}\right|} .
$$

From 29, we have

$$
\frac{|m|}{m} \tilde{b}_{i j}-\tilde{c}_{i j}=\left(\frac{\kappa}{2|m|}-\frac{\sigma \rho}{4|m|}\right) \nu_{i, j}+\frac{\sigma \rho}{2|m|} \hat{\xi}-\frac{\kappa \theta}{2|m|}=\alpha \nu_{i, j}+\beta,
$$

and from lemma 1. (57) holds true under the condition

$$
h \leq h_{2}=\min \left\{\frac{\sigma^{2} \tilde{\rho}^{2} \nu_{i}}{2 m^{2}\left|\alpha \nu_{i}+\beta\right|}, i=1,2\right\},
$$

where $\alpha$ and $\beta$ are defined in (58). Then by incorporating the conditions (56) and 59 one gets

$$
h \leq \min \left\{h_{1}, h_{2}\right\} .
$$

To guarantee the positivity of the numerical solution on boundary of the domain, it is sufficient to put condition on the coefficients $\hat{a}_{i}$ of 440 defined in 41 in terms of $h$ and $k$. This condition is

$$
k \leq \min \left\{\frac{2 h}{3 \sigma \tilde{\rho}|\hat{\xi}|}, \frac{|m| h}{3 \kappa \theta}\right\} .
$$

75 The entries of matrix $\mathcal{P}$ are nonnegative since the coefficients of the integral part of the scheme given by (28) are nonnegative. On the other hand under conditions $53,, 56),(59)$ and $(61)$, the entries of matrix $\mathcal{D}$ are also nonnegative and then the following theorem is established.

Theorem 1. With previous notation, if stepsizes $h$ and $k$ satisfy

$80 \quad C 1$.

$$
\begin{array}{ll}
\text { C1. } & h \leq \min \left\{\frac{2 \sigma \tilde{\rho} \nu_{i}}{\left|2 \hat{\xi}-\nu_{i}\right|}, \frac{\sigma^{2} \tilde{\rho}^{2} \nu_{i}}{2 m^{2}\left|\alpha \nu_{i}+\beta\right|}, i=1,2\right\} \\
\text { C2. } & k \leq \min \left\{\frac{m^{2} h^{2}}{\sigma^{2} \nu_{2}}, \frac{2 h}{3 \sigma \tilde{\rho}|\hat{\xi}|}, \frac{|m| h}{3 \kappa \theta}\right\},
\end{array}
$$

then the numerical solution $\left\{W_{i, j}^{n}\right\}$ of the scheme (28)-(41) is nonnegative. 


\subsection{Stability of the scheme}

For the sake of clarity in the presentation and as one finds many definitions of stability in the literature, we introduce the following definition

Definition 1. Let $\left\{W_{i, j}^{n}\right\}$ be a numerical solution of the PIDE computed from the scheme 281-(41) with stepsizes $h=\Delta x, h_{y}=m h$ in a rhomboid computational domain bounded by 24) and $k=\Delta \tau$ in $[0, T]$. We say that $\left\{W_{i, j}^{n}\right\}$ is strongly uniformly $\|\cdot\|_{\infty}$ stable, if the corresponding vector solution $\mathbf{W}^{n}$ given by (42) and (43) satisfies

$$
\left\|W^{n}\right\|_{\infty} \leq \Lambda\left\|W^{0}\right\|_{\infty}, \quad 0 \leq n \leq N_{\tau},
$$

where $\Lambda>0$ is independent of $n, h$ and $k$.

We begin here by providing bounds for the infinite norm of $\mathcal{D}$ and $\mathcal{P}$. From (29) and 41), under the positivity conditions of theorem 1, we have

$$
\alpha_{i, j}+\hat{\alpha}_{i, j}+\breve{\alpha}_{i, j}+\beta_{i, j}+\gamma_{i, j}=1, \quad \sum_{s=1}^{4} \hat{a}_{s}=1 .
$$

From 63 and the structure of matrices $\breve{\mathcal{C}}, \mathcal{B}$ and $\hat{\mathcal{C}}$, given by $45-47$ it follows that

$$
\|[\breve{C}(\ell) B(\ell) \hat{C}(\ell)]\|_{\infty}=\max \left\{e^{(\lambda+r) k}, 1\right\}=e^{(\lambda+r) k} .
$$

From the definition of $\mathcal{D}$ 44, property of infinite norm of the block matrices (8) and (64), one gets

$$
\|\mathcal{D}\|_{\infty}=\max \left\{1, \max _{1 \leq \ell \leq N_{x}-1}\left\{\|[\breve{C}(\ell) B(\ell) \hat{C}(\ell)]\|_{\infty}\right\}, e^{(r-q+\lambda) k}\right\}=e^{(\lambda+r) k} .
$$

In order to bound the norm of the matrix $\mathcal{P}(48)-(51)$, let $i_{m}$ be the row that coincides with the infinite norm of $\mathcal{P}$, therefore

$$
\|\mathcal{P}\|_{\infty}=\frac{5 h \lambda k}{24 \sqrt{2 \pi} \hat{\sigma} \tilde{\rho} \sigma} \sum_{\ell=0}^{N_{x} / 5-1}\left(11 g_{i_{m}, 5 \ell+1}+g_{i_{m}, 5 \ell+2}+g_{i_{m}, 5 \ell+3}+11 g_{i_{m}, 5 \ell+4}\right) .
$$

Since the right hand side of $(66)$ represents the approximation of

$$
k \lambda I_{1}=\frac{k \lambda}{\sqrt{2 \pi} \hat{\sigma} \tilde{\rho} \sigma} \int_{a}^{b} g\left(x_{i_{m}}, \phi\right) d \phi,
$$


see $(32)$, its value is given by

$$
k \lambda I_{1}=\frac{k \lambda}{2}\left(\operatorname{erf}\left(\frac{x_{i_{m}}-a+\mu \sigma \tilde{\rho}}{\sqrt{2} \sigma \tilde{\rho} \hat{\sigma}}\right)-\operatorname{erf}\left(\frac{x_{i_{m}}-b+\mu \sigma \tilde{\rho}}{\sqrt{2} \sigma \tilde{\rho} \hat{\sigma}}\right)\right) .
$$

Then for small enough $h$, we have

$$
\|\mathcal{P}\|_{\infty}<k \lambda\left(I_{1}+1\right)=k \lambda_{1}
$$

and from (42) it follows that

$$
\left\|\mathbf{W}^{n}\right\|_{\infty} \leq\left(\|\mathcal{D}\|_{\infty}+\|\mathcal{P}\|_{\infty}\right)\left\|\mathbf{W}^{n-1}\right\|_{\infty}
$$

and from (66) and (67), one gets

$$
\begin{gathered}
\frac{\left\|\mathbf{W}^{n}\right\|_{\infty}}{\left\|\mathbf{W}^{0}\right\|_{\infty}} \leq\left(e^{(r+\lambda) k}+k \lambda_{1}\right)^{n}=e^{(r+\lambda) T}\left(1+k \lambda_{1} e^{-(r+\lambda) k}\right)^{n} \\
\leq e^{(r+\lambda) T}\left(1+k \lambda_{1}\right)^{n} \leq \exp \left(\left(r+\lambda+\lambda_{1}\right) T\right) .
\end{gathered}
$$

Summarizing, according to definition (1), a conditional strong uniform stable scheme is established.

\section{Consistency}

Let us denote the local truncation error $T_{i, j}^{n}(w)$ as

$$
T_{i, j}^{n}(w)=F\left(W_{i, j}^{n}\right)-\left(L\left(w_{i, j}^{n}\right)-I\left(w_{i, j}^{n}\right)\right),
$$

90

where $w$ is the exact theoretical solution for the PIDE $[10],\left(w_{i, j}^{n}=w\left(x_{i}, y_{j}, \tau^{n}\right)\right)$, $F\left(W_{i, j}^{n}\right)=0$ represent the approximating finite difference equation $28, L(w)$ is the differential operator of 10 and $I(w)$ is the integral part given by (14). Based on the definition of consistency of [31] and [32], a numerical scheme is consistent with a PIDE if an exact theoretical solution of the PIDE approxi-

95 mates well the difference scheme as the stepsizes discretization tend to zero, i.e., the proposed scheme (28)-(41) is consistent with the PIDE (10) if $T_{i, j}^{n} \rightarrow 0$ as $h \rightarrow 0, h_{y} \rightarrow 0$ and $k \rightarrow 0$. 
Let $w$ be a continuous function of $x, y$ and $\tau$ with continuous derivatives of order four with respect to $x$ and $y$ and of order two with respect to $\tau$. By using Taylor expansion about $\left(x_{i}, y_{j}, \tau^{n}\right)$, we have

$$
\frac{w_{i, j}^{n+1}-w_{i, j}^{n}}{k}=\frac{\partial w}{\partial \tau}\left(x_{i}, y_{j}, \tau^{n}\right)+k E_{i, j}^{n}(1),
$$

where

$$
\begin{gathered}
E_{i, j}^{n}(1)=\frac{1}{2} \frac{\partial^{2} w}{\partial \tau^{2}}\left(x_{i}, y_{j}, \chi\right), n k<\chi<(n+1) k \\
\left|E_{i, j}^{n}(1)\right| \leq \frac{1}{2} \max \left\{\left|\frac{\partial^{2} w}{\partial \tau^{2}}\left(x_{i}, y_{j}, \tau\right)\right|, \tau^{n} \leq \tau \leq \tau^{n+1}\right\}=\frac{1}{2} D^{n}(1) .
\end{gathered}
$$

For the second partial derivatives with respect to the spatial variables $x$ and $y$, the Taylor's expansions are given by

$$
\begin{gathered}
\frac{w_{i+1, j}^{n}-2 w_{i, j}^{n}+w_{i-1, j}^{n}}{h^{2}}=\frac{\partial^{2} w}{\partial x^{2}}\left(x_{i}, y_{j}, \tau^{n}\right)+h^{2} E_{i, j}^{n}(2), \\
E_{i, j}^{n}(2)=\frac{1}{12} \frac{\partial^{4} w}{\partial x^{4}}\left(\chi_{1}, y_{j}, \tau^{n}\right), x_{i}-h<\chi_{1}<x_{i}+h \\
\left|E_{i, j}^{n}(2)\right| \leq \frac{1}{12} \max \left\{\left|\frac{\partial^{4} w}{\partial x^{4}}\left(x, y_{j}, \tau^{n}\right)\right|, a \leq x \leq b\right\}=\frac{1}{12} D_{j}^{n}(2),
\end{gathered}
$$

and

$$
\begin{gathered}
\frac{w_{i, j+1}^{n}-2 w_{i, j}^{n}+w_{i, j-1}^{n}}{h_{y}^{2}}=\frac{\partial^{2} w}{\partial y^{2}}\left(x_{i}, y_{j}, \tau^{n}\right)+h_{y}^{2} E_{i, j}^{n}(3), \\
E_{i, j}^{n}(3)=\frac{1}{12} \frac{\partial^{4} w}{\partial y^{4}}\left(x_{i}, \chi_{2}, \tau^{n}\right), y_{j}-h_{y}<\chi_{2}<y_{j}+h_{y}, \\
\left|E_{i, j}^{n}(3)\right| \leq \frac{1}{12} \max \left\{\left|\frac{\partial^{4} w}{\partial y^{4}}\left(x_{i}, y, \tau^{n}\right)\right|, m x_{i}-\nu_{2} \leq y \leq m x_{i}-\nu_{1}\right\}=\frac{1}{12} D_{i}^{n}(3) .
\end{gathered}
$$

The expansions for the first partial derivatives with respect to $x$ and $y$ are given by

$$
\begin{gathered}
\frac{w_{i+1, j}^{n}-w_{i-1, j}^{n}}{2 h}=\frac{\partial w}{\partial x}\left(x_{i}, y_{j}, \tau^{n}\right)+h^{2} E_{i, j}^{n}(4), \\
E_{i, j}^{n}(4)=\frac{1}{6} \frac{\partial^{3} w}{\partial x^{3}}\left(\chi_{3}, y_{j}, \tau^{n}\right), x_{i}-h<\chi_{3}<x_{i}+h,
\end{gathered}
$$




$$
\begin{gathered}
\left|E_{i, j}^{n}(4)\right| \leq \frac{1}{6} \max \left\{\left|\frac{\partial^{3} w}{\partial x^{3}}\left(x, y_{j}, \tau^{n}\right)\right|, a \leq x \leq b\right\}=\frac{1}{6} D_{j}^{n}(4) \\
\frac{w_{i, j+1}^{n}-w_{i, j-1}^{n}}{2 h_{y}}=\frac{\partial w}{\partial x}\left(x_{i}, y_{j}, \tau^{n}\right)+h_{y}^{2} E_{i, j}^{n}(5) \\
E_{i, j}^{n}(5)=\frac{1}{6} \frac{\partial^{3} w}{\partial y^{3}}\left(x_{i}, \chi_{4}, \tau^{n}\right), y_{j}-h_{y}<\chi_{4}<y_{j}+h_{y} \\
\left|E_{i, j}^{n}(5)\right| \leq \frac{1}{6} \max \left\{\left|\frac{\partial^{3} w}{\partial y^{3}}\left(x_{i}, y, \tau^{n}\right)\right|, m x_{i}-\nu_{2} \leq y \leq m x_{i}-\nu_{1}\right\}=\frac{1}{6} D_{i}^{n}(5)
\end{gathered}
$$

On the other hand for the integral part, there are two error sources; the first coming from the truncation of improper integral into a bounded one $(a, b)$ and the second coming from the numerical approximation of the finite integral using the four point open type formula. Let $\mathcal{T}_{i, j}^{n}(w)$ denote the total truncation error for the integral part such that

$$
\begin{aligned}
\mathcal{T}_{i, j}^{n}(w)=I\left(w_{i, j}^{n}\right)-\hat{\lambda} J_{i, j}^{n} & =\left(I\left(w_{i, j}^{n}\right)-I_{a b}\left(w_{i, j}^{n}\right)\right)+\left(I_{a b}\left(w_{i, j}^{n}\right)-\hat{\lambda} J_{i, j}^{n}\right) \\
& =\mathcal{H}_{i, j}^{n}(w)+\mathcal{Y}_{i, j}^{n}(w)
\end{aligned}
$$

where $I_{a b}(w)=\frac{\lambda}{\sqrt{2 \pi} \hat{\sigma} \tilde{\rho} \sigma} \int_{a}^{b} g(x, \phi) w(x, y+m(\phi-x), \tau) d \phi$, the truncation er100 ror $\mathcal{H}_{i, j}^{n}(w)=I(w)-I_{a b}(w)$ and the error due to the numerical integration $\mathcal{Y}_{i, j}^{n}(w)=I_{a b}(w)-\hat{\lambda} J_{i, j}^{n}$.

According to Briani et. al. [33, since the integral part contains the Gaussian function, then the absolute value of $\mathcal{H}_{i, j}^{n}(w)$ can be controlled using a tolerance parameter error $\varepsilon>0$ by choosing

$$
b=\sqrt{-2 \hat{\sigma}^{2} \ln (\varepsilon \hat{\sigma} \sqrt{2 \pi})}, a=-b .
$$

Furthermore, due to the symmetric property of the probability measure of Gaussian distribution, one can assume that the option price $w$ satisfies the Lipschitz condition with respect to the spacial variables, then one has [3],

$$
\left|\mathcal{H}_{i, j}^{n}(w)\right|<2 \hat{\sigma}^{2} \varepsilon
$$


Finally, from [24, 95],

$$
\left|\mathcal{Y}_{i, j}^{n}(w)\right| \leq \frac{90 h^{4}}{144} D_{i, j}^{n}(6)
$$

where

$$
D_{i, j}^{n}(6)=\max \left\{\left(w\left(\phi, y_{j}+m\left(\phi-x_{i}\right), \tau^{n}\right)\right)^{(4)}, a \leq \phi \leq b\right\}
$$

and the fourth derivative of the function $w\left(\phi, y_{j}+m\left(\phi-x_{i}\right), \tau^{n}\right)$ is taken with respect to $\phi$. Hence the total error for the integral part $\left|\mathcal{T}_{i, j}^{n}\right|$ satisfies

$$
\left|\mathcal{T}_{i, j}^{n}\right|<2 \hat{\sigma}^{2} \varepsilon+\frac{90 h^{4}}{144} D_{i, j}^{n}(6) .
$$

From (72), 744, 776, 778$),(80), 820$ and (71), the local truncation error has the following form

$$
\begin{gathered}
T_{i, j}^{n}=k E_{i, j}^{n}(1)-\frac{\tilde{\rho}^{2} \nu_{i, j} \sigma^{2}}{2}\left(h^{2} E_{i, j}^{n}(2)+m^{2} h^{2} E_{i, j}^{n}(3)\right)-\hat{\delta}_{i, j} h^{2} E_{i, j}^{n}(4) \\
-\tilde{\delta}_{i, j} m^{2} h^{2} E_{i, j}^{n}(5)-\mathcal{T}_{i, j}^{n}(w),
\end{gathered}
$$

where $\hat{\delta}_{i, j}$ and $\tilde{\delta}_{i, j}$ correspond to expressions appearing in 12 when replacing $\nu$ by $\nu_{i, j}$. Finally, from (73), 75), 777, 879, 81), 87) and 88, we have

$$
\begin{aligned}
\left|T_{i, j}^{n}\right| \leq \frac{k}{2} D^{n}(1)+\left|\frac{\tilde{\rho}^{2} \nu_{i, j} \sigma^{2}}{24}\right| & \left(D_{j}^{n}(2)+m^{2} D_{i}^{n}(3)\right) h^{2}+\left(\left|\hat{\delta}_{i, j}\right| D_{j}^{n}(4)+m^{2}\left|\tilde{\delta}_{i, j}\right| D_{i}^{n}(5)\right) \frac{h^{2}}{6} \\
& +\frac{90 h^{4}}{144} D_{i, j}^{n}(6)+2 \hat{\sigma}^{2} \varepsilon
\end{aligned}
$$

Therefore

$$
\left|T_{i, j}^{n}\right| \leq \mathcal{O}(k)+\mathcal{O}\left(h^{2}\right)+\mathcal{O}(\varepsilon) .
$$

Summarizing, the consistency for the scheme is established.

\section{Numerical Examples}

After removing the mixed derivative of the PIDE (1) for Bates model, a finite difference scheme has been constructed to obtain a numerical approximation for the option price. Furthermore, the positivity conditions are provided, 
also stability and consistency have been studied. In this section, several examples are provided to study the behavior of the option price obtained by the proposed scheme using Matlab. The used computer has Microprocessor $3.4 \mathrm{GHz}$ Intel Core i7. The following example reveals the importance of the positivity conditions (60) and (61) on the stepsizes $h$ and $k$.

Example 1. Consider an European call option under Bates model with the following parameters $T=0.5, E=100, r=0.05, q=0, \theta=0.05, \kappa=2.5$, $\sigma=0.25, \hat{\sigma}=0.7, \mu=0.5, \lambda=0.2 \nu_{1}=0.1, \nu_{2}=1$ and $\rho=-0.5$ with a tolerance error $\varepsilon=10^{-3}$. In Figure 2, the solid curve represents the option price as a function of the underlying asset $S$ when the positivity conditions hold for $\left(N_{x}, N_{y}, N_{\tau}\right)=(100,45,150)$ corresponding to $h=0.05$ and $k=0.0033$, while the dashed curve represents the option price when the positivity conditions are broken for $\left(N_{x}, N_{y}, N_{\tau}\right)=(100,45,50)$ corresponding to $h=0.05$ and $k=0.01$.

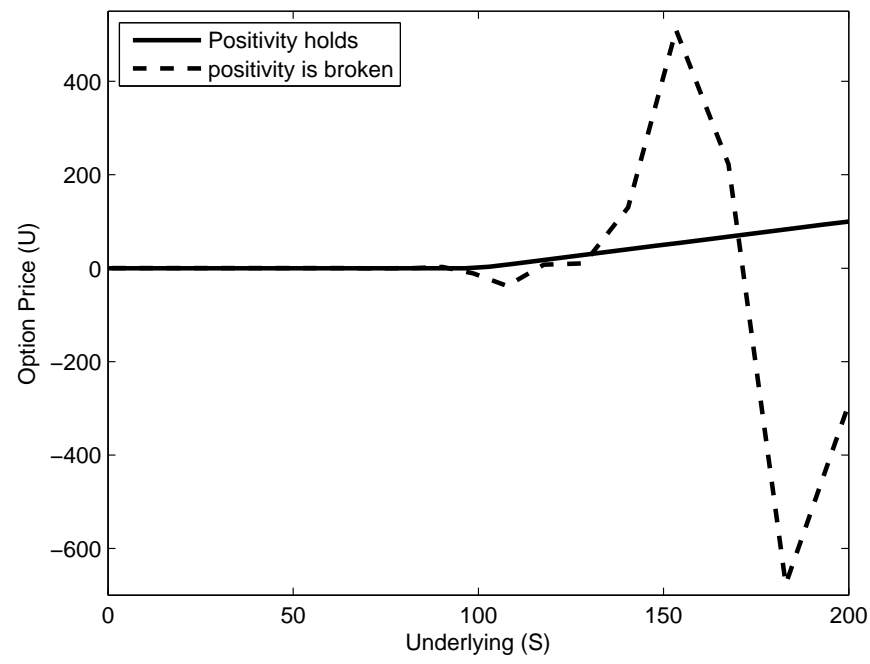

Fig. 2. The effect of positivity conditions on the option price $U$

The next example investigates the associated error for the scheme 28 - 41 when $\lambda=0$, i.e., for European option under Heston model. Considering the strike price $E=100$, the numerical solutions for the set of underlying assets $\mathcal{S}=$ 
$\{80,90,100,110,120\}$ are obtained. In order to evaluate the error, a Matlab code for the closed form solution is used [34] obtaining the set of corresponding reference option price values $\mathcal{U}=\{0.207581,4.889877,10.488226,16.503506,22.856611\}$. The root mean square relative error (RMSRE) is calculated based on the equation

$$
\text { RMSRE }=\sqrt{\frac{1}{5} \sum_{i=1}^{5}\left(\frac{\mathcal{U}\left(\mathcal{S}_{i}, \nu_{0}, T\right)-U\left(\mathcal{S}_{i}, \nu_{0}, T\right)}{\mathcal{U}\left(\mathcal{S}_{i}, \nu_{0}, T\right)}\right)^{2}},
$$

where $U\left(\mathcal{S}_{i}, \nu_{0}, T\right)$ is the numerical solution at spot variance $\nu_{0}=0.4$.

Example 2. Here the parameters are chosen as follows $T=0.5, E=100$, ${ }_{125} r=0.05, q=0, \theta=0.05, \kappa=2, \sigma=0.3$, and $\rho=-0.5$. The computational domain is $[a, b]=[-0.5,1.5], \nu_{1}=0.1$ and $\nu_{2}=1$. Table 1 exhibits the variation of RMSRE for several values of $N_{\tau}$ while $N_{x}=70$ and $N_{y}=16$, the numerical order of error and CPU time in seconds.

\begin{tabular}{llll}
\hline$N_{\tau}$ & RMSRE & Ratio & CPU (sec) \\
\hline 200 & $1.764 \times 10^{-3}$ & - & 1.01 \\
130 & $9.387 \times 10^{-4}$ & 1.88 & 1.05 \\
400 & $4.581 \times 10^{-4}$ & 2.05 & 1.17 \\
1600 & $2.371 \times 10^{-4}$ & 1.93 & 1.19 \\
3200 & $1.191 \times 10^{-4}$ & 1.99 & 1.32 \\
\hline
\end{tabular}

In Table 2, the variation of error due to the change of the spatial step sizes, while $N_{\tau}=500$ has been studied.

\begin{tabular}{llll}
\hline$\left(N_{x}, N_{y}\right)$ & RMSRE & Ratio & CPU $(\mathrm{sec})$ \\
\hline$(40,9)$ & $4.166 \times 10^{-3}$ & - & 0.11 \\
$(60,14)$ & $2.986 \times 10^{-3}$ & 1.395 & 0.71 \\
$(80,18)$ & $9.367 \times 10^{-4}$ & 3.188 & 2.52 \\
$(100,23)$ & $3.861 \times 10^{-4}$ & 2.426 & 7.476 \\
$(120,27)$ & $9.287 \times 10^{-5}$ & 4.157 & 19.53 \\
\hline
\end{tabular}


The aim of the last example is to study the variation of the resultant error for European option under Bates model.

Example 3. The parameters are selected as follows $T=0.5, E=100, r=0.05$, $q=0, \theta=0.05, \kappa=2.0, \sigma=0.3, \hat{\sigma}=0.35, \mu=-0.5, \lambda=0.2$ and $\rho=-0.5$ with a tolerance error $\varepsilon=10^{-4}$. The boundary points $a$ and $b$ of the spatial computational domain are obtained from (83), while $\nu_{1}=0.1$ and $\nu_{2}=1$. Table 3 shows the variation of the RMSRE for several values of the time step sizes, for fixed $N_{x}=70$ and $N_{y}=35$, with respect to reference values computed at $\left(N_{x}, N_{y}, N_{\tau}\right)=(500,146,7000)$.

\begin{tabular}{llll}
\hline$N_{\tau}$ & RMSRE & Ratio & CPU (sec) \\
\hline 500 & $2.485 \times 10^{-3}$ & - & 6.66 \\
1000 & $1.322 \times 10^{-3}$ & 1.88 & 6.94 \\
2000 & $6.429 \times 10^{-4}$ & 2.06 & 7.28 \\
4000 & $3.296 \times 10^{-4}$ & 1.95 & 7.69 \\
8000 & $1.569 \times 10^{-4}$ & 2.10 & 7.91 \\
\hline \multicolumn{5}{c}{}
\end{tabular}

The variation of error due to the change of the spatial step sizes, while $N_{\tau}=500$ has been presented in Table 4.

\begin{tabular}{llll}
\hline$\left(N_{x}, N_{y}\right)$ & RMSRE & Ratio & CPU (sec) \\
\hline$(40,20)$ & $1.526 \times 10^{-2}$ & - & 0.32 \\
$(60,30)$ & $3.459 \times 10^{-3}$ & 4.412 & 1.83 \\
$(80,40)$ & $9.271 \times 10^{-4}$ & 3.371 & 6.95 \\
$(100,50)$ & $3.589 \times 10^{-4}$ & 2.583 & 19.64 \\
$(120,60)$ & $8.473 \times 10^{-5}$ & 4.236 & 46.72 \\
\hline \multicolumn{5}{c}{ Table (4): The associated RMSRE for different values of $\left(N_{x}, N_{y}\right)}$. \\
\end{tabular}




\section{Acknowledgements}

This work has been partially supported by the European Union in the FP7PEOPLE-2012-ITN program under Grant Agreement Number 304617 (FP7 Marie Curie Action, Project Multi-ITN STRIKE-Novel Methods in Computational Finance) and the Ministerio de Economía y Competitividad Spanish grant MTM2013-41765-P.

\section{References}

[1] F. Black, M. Scholes, The pricing of options and corporate liabilities, J. Political Economy. 81 (1973) 637-654.

[2] R. C. Merton, Option pricing when underlying stock returns are discontinuous, J. Financial Economics. 3 (1976) 125-144.

[3] S. G. Kou A jump diffusion model for option pricing, Management Science. 48 (2002) 1086-1101.

[4] J. Hull, A. White, The pricing of options on assets with stochastic volatilities, The Journal of Finance. 42 (1987) 281-300.

[5] S. L. Heston, A closed-form solution for options with stochastic volatility with applications to bond and currency options, The Review of Financial Studies. 6 (1993) 327-343.

[6] A. Pascucci, PDE and martingale methods in option pricing, volume 2 of Bocconi \& Springer Series. Springer, Milan, 2011.

175 [7] R. Cont, P. Tankov, Financial Modelling with Jump Processes, Chapman \& Hall/CRC Financial Mathematics Series, Chapman \& Hall/CRC, Boca Raton, FL, 2004.

[8] J. Toivanen, A Componentwise Splitting Method for Pricing American Options under the Bates Model, Applied and Numerical Partial Differential 
in a Multidisciplinary Context, W. Fitzgibbon, Yu. Kuznetsov, P. Neittaanmaki, J. Periaux, O. Pironneau (eds.), Computational Methods in Applied Sciences, Springer, 15 (2010) 213-227.

[9] L. O. Scott, Pricing stock options in a jump-diffusion model with stochastic volatility and interest rates: applications of Fourier inversion methods, Mathematical Finance 7 (1997) 413-426.

[10] G. Bakshi, C. Cao and Z. Chen, Empirical performance of alternative option pricing models, The Journal of Finance, LII5 (1997) 2003-2049.

[11] L. Feng and V. Linetsky, Pricing options in jump-diffusion models an extrapolation approach, Operations Research, 56 (2008) 304-325.

[12] D. S. Bates, Jumps and stochastic volatility: Exchange rate processes implicit Deutsche mark options, Review Financial Studies, 9 (1996) 69-107.

[13] C. Chiarella, B. Kang, G. H. Mayer, and A. Ziogas, The evaluation of American option prices under stochastic volatility and jump-diffusion dynamics using the method of lines. Research Paper Quantitative Finance Research Centre University of Technology, Sydney, 219 (2008) 1-43.

[14] D. J. Duffy, Finite Difference Methods in Financial Engineering: a Partial Differential Approach, John Wiley \& Sons Ltd, The Atrium, Southern Gate, Chichester, West Sussex PO19 8SQ, England, 2006.

[15] P. Wilmott, J. Dewynne and S. Howison, Option Pricing: Mathematical Models and Computation, Oxford Financial press, 1998.

[16] G. H.Meyer and J. van der Hoek, The Evaluation of American Options with the Method of Lines, Advances in Futures and Options Research 9 (1997) 265-285.

[17] S. Salmi, J. Toivanen and L. Von Sydow, Iterative methods for pricing American options under the bates model, Procedia Computer Science, 18 (2013) 1136-1144. 
[18] R. Rannacher, Finite element solution of diffusion problems with irregular data, Numer. Math. 43 (1984) 309-327.

[19] R. Zvan, P. A. Forsyth, and K. R. Vetzal, Negative coefficients in two-factor option pricing models, Journal of Computational Finance, 7 (2003) 37-73.

[20] R. Company, L. Jódar, M. Fakharany and M.-C. Casabán, Removing the correlation term in the option pricing Heston model: numerical analysis and computing, Abstract and Applied Analysis, 2013 (2013) 1-11.

[21] B. Düring and M. Fournié, High-order compact finite difference scheme for option pricing in stochastic volatility models, Journal of Computational and Applied Mathematics, 236 (2012) 4462-4473.

[22] B. Düring, M. Fournié and C. Heuer, High-order compact finite difference schemes for option pricing in stochastic volatility models on non-uniform grids, Journal of Computational and Applied Mathematics 271 (2014) 247266.

[23] N. Clarke and K. Parrott, Multigrid for american option pricing with stochastic volatility, Applied Mathematical Finance, 6 (3), (1999), 177-195.

[24] P. J. Davis, P. Rabinowitz, Methods of Numerical Integration, Second Edition, Academic Press, New York, USA, 1984.

[25] G. H. Golub and C. F. Van Loan, Matrix Computations, third edition, Johns Hopkins University Press, 1996.

[26] L. C, Andrews, Special Functions for Engineers and Applied Mathematicians, Macmillan Publishing Company, New York, 1985.

${ }_{230}^{23}$ [27] P. R. Garabedian, Partial Differential Equations, AMS Chelsea Pubs. Co., 1998.

[28] R. Kangro, R. Nicolaides, Far field boundary conditions for Black-Scholes equations, SIAM Journal on Numerical Analysis 38 (4) (2000) 1357-1368. 
[29] M. Ehrhardt, R. Mickens, A fast, stable and accurate numerical method for the Black-Scholes equation of american options, International Journal of Theoretical and Applied Finance 11 (2008) 471-501.

[30] J. W. Thomas, Numerical Partial Differential Equations Finite Difference Methods. Springer, 1995.

[31] G. D. Smith, Numerical Solution of Partial Differential Equations: Finite Difference Methods (3rd ed.), Clarendon Press, Oxford, UK, 1985.

[32] P. Linz, Analytic and Numerical Methods for Volterra Equations, SIAM, Philadelphia, USA, 1985.

[33] M. Briani, C. La Chioma and R. Natalini,Convergence of numerical schemes for viscosity solutions to integro-differential degenerate parabolic problems arising in financial theory. Numer. Math., 98 (4) (2004) 607-646.

[34] W. Poklewski-Koziell, "Stochastic Volatility Models: Calibration, Pricing and Hedging", University of the Witwatersrand, South Africa, MSc thesis 2012. 